\title{
Modeling wave and wind climate effects on tidal sand wave dynamics: A North Sea case study
}

\author{
G.H.P. Campmans*, P.C. Roos, E.P.W.J. Schrijen, S.J.M.H. Hulscher \\ Water Engineering and Management, PO Box 217, 7500AE, Enschede, the Netherlands
}

\section{A R T I C L E I N F O}

\section{Keywords:}

Tidal sand waves

Storm effects

Linear stability analysis

Wave and wind climate

Idealized modeling

Morphodynamics

\begin{abstract}
A B S T R A C T
To obtain site-specific wave and wind climate averaged sand wave dynamics, we combine an idealized linear stability model with 20 years of wave and wind data taken from the Euro Platform in the North Sea. The model output results in a wave and wind climate-averaged growth and migration rate. The results show that waves and wind affect particularly migration and to a much smaller extent the growth rate. Seasonal variations in wave and wind conditions during winter and summer periods result in seasonal variations in sand wave dynamics, in particular during winter the migration rate is larger, the growth rates are lower and the preferred wavelength is larger compared to summer. Medium wave and wind conditions are responsible for two thirds of the migration rate, while these conditions occur roughly only one third of the time. Extreme wave and wind conditions result in only a moderate contribution to the migration rate. Furthermore, we see a seasonal variation in migration as well as reasonable correlation with observed migration rates for the intervals between surveys in the period 1996-2010. Our work shows that storms are able to affect sand wave migration, and cause variability in migration rate.
\end{abstract}

\section{Introduction}

Tidal sand waves are rhythmic bed forms observed in tidally dominated shallow seas all around the world. They have typical wavelengths of hundreds of meters and heights of several meters (Terwindt, 1971; Van Dijk and Kleinhans, 2005, also see Fig. 1a). These large bed forms are dynamic, e.g. showing migration and growth. Because of their dynamical behavior in combination with their large dimensions, they interfere with various human activities in shallow seas. For example, sand waves may pose a hazard to navigation, pipelines, cables as well as the foundation of wind farms and gas/oil platforms (Németh et al., 2003). Therefore, an accurate understanding of sand wave dynamics is required.

Sand waves have been studied both using observations and from a modeling perspective. Hulscher (1996) explained sand waves as a free instability of the sandy seabed subject to tidal flow. Tidally averaged circulation cells tend to move sediment towards the crests, while gravity favors sediment transport towards the troughs. Observational studies show that storms, which occur on a much more irregular basis compared to the tidal flow, also affect sand wave dynamics. In particular, sand wave heights are reduced during stormy periods (Terwindt, 1971; Houthuys et al., 1994; Van Dijk and Kleinhans, 2005) and migration speed or even direction may change (Harris, 1989; Fenster et al., 1990; Le Bot et al., 2000).

To better understand sand wave dynamics, various physical processes that affect their dynamics have been investigated using processbased modeling approaches. As shown by Hulscher (1996), small-amplitude sand wave dynamics can be investigated using linear stability analysis. It produces growth and migration rates as a function of the topographic wave vector $\left(k_{x}, k_{y}\right)$, which is related to wavelength and crest orientation. If the growth rates are negative for all $\left(k_{x}, k_{y}\right)$, i.e. the perturbations decay, the flat seabed is stable. If some mode grows, the seabed is unstable, and the fastest growing mode (FGM) is assumed to be the dominant bed form (Dodd et al., 2003). The FGM properties have successfully been used to predict sand wave occurrence (Hulscher and Van den Brink, 2001) and variations (Van Santen et al., 2011). By extending Hulscher's approach, sand wave migration has been explained by pressure- or wind-driven currents (Németh et al., 2002) and by tidal asymmetry (Besio et al., 2004). Other studies investigated the effects of grain-size variation (Roos et al., 2007; Van Oyen and Blondeaux, 2009), benthos (Borsje et al., 2009) and non-erodible bed layers (Blondeaux et al., 2016). Blondeaux and Vittori (2016) successfully compared modeled sand waves with observations (Menninga, 2012). They included the spring-neap cycle, a residual current and a single wind wave condition. Inspired by the storm-related observations mentioned above, Campmans et al. (2017) developed a linear stability model to

\footnotetext{
* Corresponding author.

E-mail address: g.h.p.campmans@utwente.nl (G.H.P. Campmans).
} 
(a)

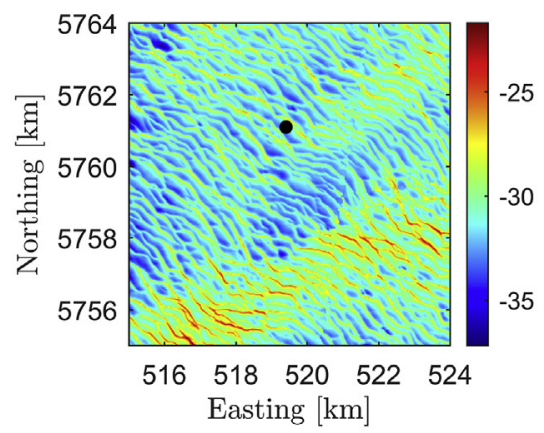

(b)

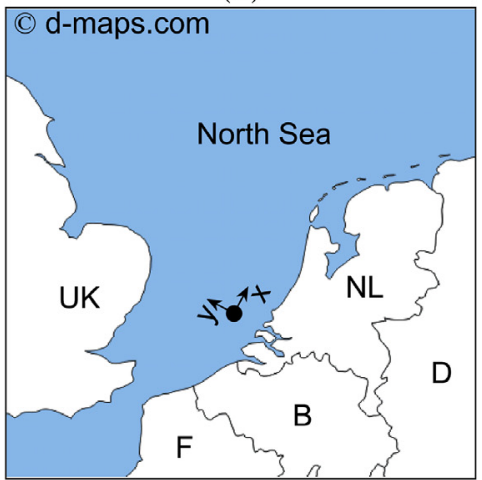

(c)

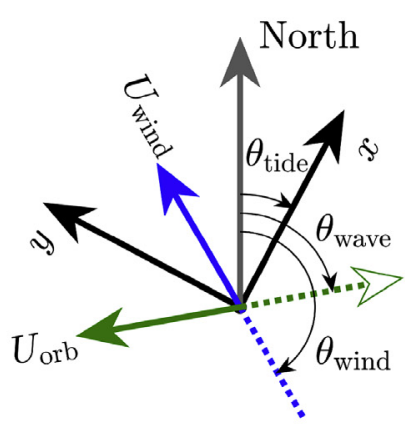

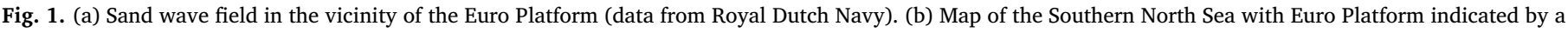

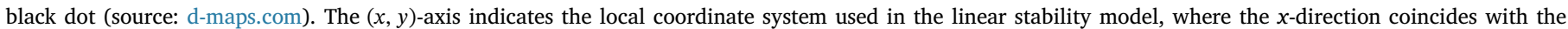
principal tidal flow direction. (c) Definition sketch defining wave angle and wind angle clockwise w.r.t. north.

systematically investigate the effect of wind-driven flow and wind waves on sand wave dynamics. Both waves and wind are potentially able to reduce the growth rate of sand waves. Wind-driven flow induces sand wave migration and wind waves enhance migration already occurring due to other processes. Importantly, the results by Campmans et al. (2017) were obtained by studying the various wave and wind conditions in isolation; their weighted effects in wave and wind climate averaged sense were not taken into account. Strictly speaking, linear stability analysis is only valid for infinitesimally small amplitudes. However, applying it in practice with a finite amplitude gives a reliable approximation (e.g., up to $1 \mathrm{~m}$, as illustrated in Fig.11 in Campmans et al., 2018). Linear stability models typically are computationally fast (seconds), which makes them suitable to explore the parameter space in detailed sensitivity studies.

Capturing finite amplitude sand wave dynamics including equilibrium shape and height requires a nonlinear modeling approach (Németh et al., 2007; Van den Berg et al., 2012; Van Gerwen et al., 2018; Campmans et al., 2018). However, these models are computationally heavy (hours/days, or even longer), which puts limits to their applicability.

As outlined above, both linear and nonlinear model approaches have their own benefits and weaknesses. Ideally they should be used in combination, such that computationally heavy nonlinear models are used with parameters obtained from the fast linear stability model.

The aim of this work is to determine the effects of a wave and wind climate on sand wave dynamics at a typical North Sea location. Restricting to small-amplitude dynamics, we specifically address the following three research questions:

- How does the North Sea wave and wind climate affect the sand wave growth and migration rates?

- Which storm conditions have the largest impact on sand wave dynamics, e.g. the more moderate, frequent conditions or the extreme, rare conditions?

- How do the properties of the fastest growing mode (growth rate, migration rate, wavelength) vary seasonally, i.e. distinguishing the rougher winter periods from milder summer periods?

To answer these questions, we process time series of observations into a wave and wind climate, which is then used as input for the linear stability model by Campmans et al. (2017). The model output results in a wave and wind climate-averaged growth and migration rate as function of $k_{x}$ and $k_{y}$. This requires a statistical approach in which the joint probability of wave and wind parameters is taken into account in the climate-averaged model outcome. As location we choose the Euro Platform, which is an offshore structure to aid navigation and equipped with wave and wind measurement instruments. It is located in a sand wave field at approximately $50 \mathrm{~km}$ west of the port of Rotterdam (Fig. 1). The innovation of this work is that we combine modeled wave and wind effects with the joint probability of wave and wind conditions obtained from observational data in order to generate site-specific results.

The paper is structured as follows. Section 2 explains how wave and wind data are used together with the linear stability model and provides values of the model parameters at the research location. In Section 3 the resulting effect of the wave and wind climate on sand wave dynamics is shown and the contributions of various wave and wind conditions are explained. Finally, Sections 4 and 5 contain the discussion and conclusion, respectively.

\section{Methods}

\subsection{Site-specific conditions at the Euro Platform}

The average wavelength and wave height of the sand waves as presented in Fig. 1 are $230 \mathrm{~m}$ (ranging from approximately $110-450 \mathrm{~m}$ ) and $2.7 \mathrm{~m}$ (ranging from approximately $0.1-7.6 \mathrm{~m}$ ), respectively. Damen et al. (2018) performed an extensive data analysis study to systematically investigate correlations between sand wave characteristics and environmental properties across the entire Dutch continental shelf, including our case study area. This work identified areas that are bed load or suspended load dominated. Since our study location is in a bed load dominated region (see Fig. 8A in Damen et al., 2018), we decided not to include suspended load sediment transport. These model parameters include site-specific values of, among others, a sediment grain size of $393 \mu \mathrm{m}, 0.2$ tidal ellipticity, a tidal flow oriented at $28^{\circ}$ w.r.t. north, and a water depth of $31 \mathrm{~m}$ (Damen et al., 2018). In the same area Menninga (2012) analyzed sand wave dynamics, including migration. Our model findings will be compared with their observed sand wave migration.

\subsection{Wave and wind data at the Euro Platform}

At the Euro Platform hourly wave and wind measurement data are available from Rijkswaterstaat (n.d.) and KNMI (n.d.), respectively. The hourly measurements contain wave height $H_{\mathrm{w}}$, wave period $T_{\mathrm{w}}$, wave direction $\theta_{\text {wave }}$, wind speed $U_{\text {wind }}$ and wind direction $\theta_{\text {wind }}$ during the period 1996-2017, of which a three-month section is shown in Fig. 2.

The wave and wind condition vector $\xi$ is

$\xi=\left(U_{\text {wind }}, \theta_{\text {wind }}, U_{\text {orb }}, \theta_{\text {wave }}\right)$,

which contains the wind speed $U_{\text {wind }}$, wind direction $\theta_{\text {wind }}$, near-bed wave orbital velocity amplitude $U_{\text {orb }}$ and wave direction $\theta_{\text {wave }}$. The wave and wind angles $\theta_{\text {wave }}$ and $\theta_{\text {wind }}$ (i.e., the directions from which they 

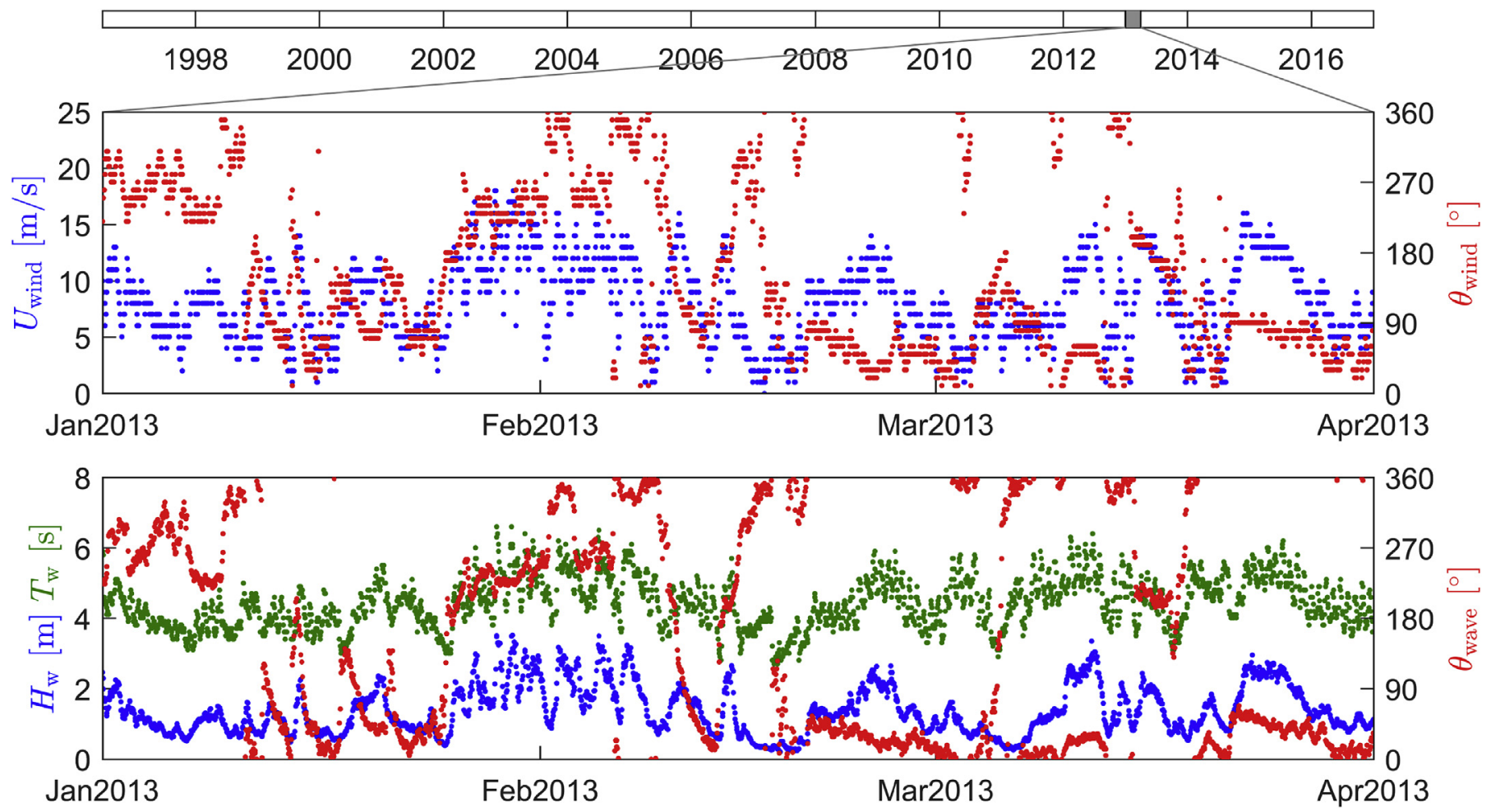

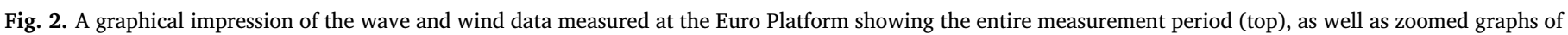
wind speed $U_{\text {wind }}$ and angle $\theta_{\text {wind }}$ (middle) and wave height $H_{\mathrm{w}}$, period $T_{\mathrm{w}}$ and angle $\theta_{\text {wave }}$ (bottom) for the period January 1 st - April 1 st, 2013 .

Table 1

Bin bounds for each of the four wave and wind parameters, leading to a total of $12^{4}=20,736$ bins.

\begin{tabular}{llll}
\hline$U_{\text {wind }}[\mathrm{m} / \mathrm{s}]$ & $\theta_{\text {wind }}\left[{ }^{\circ}\right]$ & $U_{\text {orb }}[\mathrm{m} / \mathrm{s}]$ & $\theta_{\text {wave }}\left[^{\circ}\right]$ \\
\hline 0.00 & 28 & 0.000 & 13 \\
2.08 & 58 & 0.042 & 28 \\
4.17 & 88 & 0.083 & 43 \\
6.25 & 118 & 0.125 & 58 \\
8.33 & 148 & 0.167 & 73 \\
10.42 & 178 & 0.208 & 88 \\
12.50 & 208 & 0.250 & 103 \\
14.58 & 238 & 0.292 & 118 \\
16.67 & 268 & 0.333 & 133 \\
18.75 & 298 & 0.375 & 148 \\
20.83 & 328 & 0.417 & 163 \\
22.92 & 358 & 0.458 & 178 \\
25.00 & 28 & 0.500 & 193 \\
\hline
\end{tabular}

approach) are measured clockwise with respect to north, see Fig. 1c. The near-bed orbital velocity amplitudes are computed using linear wave theory (e.g., Mei, 1989) according to

$U_{\text {orb }}=\frac{\pi H_{\mathrm{w}}}{T_{\mathrm{w}} \sinh k_{\mathrm{w}} h}$,

where $H_{\mathrm{w}}$ is the wave height, $T_{\mathrm{w}}$ the wave period, $h$ the water depth and $k_{\mathrm{w}}$ the wave number determined by the dispersion relation

$\left(\frac{2 \pi}{T_{\mathrm{w}}}\right)^{2}=g k_{\mathrm{w}} \tanh \left(k_{\mathrm{w}} h\right)$.

Here $g$ is the gravitational acceleration constant. Importantly, Eq. (2) enables us to combine two parameters (wave period $T_{\mathrm{w}}$ and wave height $H_{\mathrm{w}}$ ) into one single parameter $U_{\mathrm{orb}}$, thus reducing the dimension of the parameter space from five to four. This speeds up the computations.

\subsection{Joint wave and wind probability density function}

Using the data, a joint probability density function of the occurrence of wave and wind parameter combinations $\xi$ is determined. The data combinations, $\xi$, are binned into $M=12^{4}$ equally spaced bins. For all bins the probability of the wave and wind conditions is computed using the hourly measured wave and wind data, according to

$P\left(\xi_{\text {lower }}, \boldsymbol{\xi}_{\text {upper }}\right)=\frac{1}{N} \sum_{n=1}^{N} \eta\left(\xi_{n}, \xi_{\text {lower }}, \boldsymbol{\xi}_{\text {upper }}\right)$.

Here $P\left(\xi_{\text {lower }}, \xi_{\text {upper }}\right)$ is the probability of wave conditions $\xi$ to be inside the bin bounded by lower and upper interval limits $\xi_{\text {lower }}$ and $\xi_{\text {upper}}$, respectively. Furthermore, $N$ is the total number of hourly wave and wind data points, here $N=169,657, \xi_{n}$ represents the measured wave and wind conditions for the $n^{\text {th }}$ data point, see Eq. (1). Finally, $\eta\left(\boldsymbol{\xi}_{n}, \boldsymbol{\xi}_{\text {lower }}, \boldsymbol{\xi}_{\text {upper }}\right)$ is a function that returns one if the measurement $\boldsymbol{\xi}_{n}$ is inside the bin and zero otherwise. The bin bounds applied in this study are shown in Table 1.

The bin bounds for the wave and wind angles are chosen such that the bins are defined symmetrically around the tidal current direction $\left(\theta_{\text {tide }}=28^{\circ}\right.$ w.r.t. north, see Fig. $1 \mathrm{c}$.

\subsection{Outline of linear stability model}

The sand wave model is based on the morphodynamic loop and consists of three parts: (1) The hydrodynamics are described by the 3D shallow water equations which describe tidal and wind-driven flow. (2) Sediment transport is modeled by a bed load formula. Here wave stirring is taken into account. (3) The seabed evolution satisfies the Exner equation, which describes sediment conservation.

Consider a sinusoidal seabed perturbation (representing a sand wave) characterized by topographic wave vector $\left(k_{x}, k_{y}\right)$, with magnitude $k=\left(k_{x}^{2}+k_{y}^{2}\right)^{1 / 2}$. The model solution describes exponential growth (or decay) and migration of this individual mode: 
Table 2

Overview of model parameters.

\begin{tabular}{llll}
\hline Model parameter & Symbol & Reference value $^{\dagger}$ & Unit \\
\hline Water depth & $H$ & 31 & $\mathrm{~m}$ \\
Tidal current velocity (M2) & $U$ & 0.5 & $\mathrm{~m} \mathrm{~s}^{-1}$ \\
Tidal frequency (M2) & $\sigma$ & $1.41 \cdot 10^{-4}$ & $\mathrm{rad} \mathrm{s}^{-1}$ \\
Tidal angle (M2) & $\theta_{\text {tide }}$ & 28 & $\circ$ \\
Wave friction factor & $f_{\mathrm{w}}$ & 0.1 & - \\
Gravitational acceleration & $g$ & 9.81 & $\mathrm{~m} \mathrm{~s}^{-2}$ \\
Vertical eddy viscosity & $A_{\mathrm{v}}$ & 0.04 & $\mathrm{~m}^{2} \mathrm{~s}^{-1}$ \\
Slip parameter & $S$ & 0.01 & $\mathrm{~m} \mathrm{~s}^{-1}$ \\
Slope correction factor & $\lambda$ & 1.5 & - \\
Tidal ellipticity (M2) & $\varepsilon_{\mathrm{M} 2}$ & 0.2 & - \\
Sediment grain size & $d$ & 393 & $\mu \mathrm{m}$ \\
Bed load exponent & $\beta$ & 1.5 & - \\
Bed load coefficient & $\alpha$ & $1.51 \cdot 10^{-5}$ & $\mathrm{~m}^{\beta+2} \mathrm{~s}^{2 \beta-1} \mathrm{~kg}^{-\beta}$ \\
\hline
\end{tabular}

$h(x, y, t)=h_{\text {init }} \exp (\omega t) \cos \left(k_{x} x+k_{y} y-k c_{\text {mig }} t\right)$.

Here, $h_{\text {init }}$ is the initial amplitude of the perturbation, $\omega$ the growth rate and $c_{\text {mig }}$ the migration rate (in the direction orthogonal to the sand wave crests). These growth and migration rates depend on $\left(k_{x}, k_{y}\right)$. As pointed out in the introduction, if the growth rate is negative for all $\left(k_{x}, k_{y}\right)$ the flat seabed is stable. Otherwise, it is unstable and the perturbation with the maximum growth rate is termed the 'fastest growing mode' (FGM).

Here we use the linear stability model to analyze the stability of a flat seabed under different wave and wind conditions. This can be written as

$\omega=\omega\left(k_{x}, k_{y}, U_{\text {wind }}, \theta_{\text {wind }}, U_{\text {orb }}, \theta_{\text {wave }}\right)$,

$c_{\text {mig }}=c_{\text {mig }}\left(k_{x}, k_{y}, U_{\text {wind }}, \theta_{\text {wind }}, U_{\text {orb }}, \theta_{\text {wave }}\right)$.

The model parameters, other than wave and wind conditions, are presented in Table 2. For a detailed model description we refer to Campmans et al. (2017).

\subsection{Combining model results with wave and wind probability}

In the linear regime, the sequence in which the various wave and wind conditions occur, is irrelevant. This property enables us to compute growth and migration rates that are averaged over the combined wave and wind climate. These are termed the climate-averaged growth and migration rates.

For each bin of wave and wind conditions for which the weighting factor is nonzero, the linear stability model is applied with the wave and wind conditions coinciding with the bin centers $\bar{\xi}=\frac{1}{2}\left(\xi_{\text {lower }}+\xi_{\text {upper }}\right)$.

The climate-averaged growth and migration rates, $\langle\omega\rangle$ and $\left\langle c_{\text {mig }}\right\rangle$ respectively, are computed by multiplying the linear stability model output for the bin-centered wave and wind conditions with the corresponding probability $P_{m}$ of those conditions, and summing over all $M=12^{4}$ bins:

$\langle\omega\rangle=\sum_{m=1}^{M} P_{m} \omega_{m}$

$\left\langle c_{\mathrm{mig}}\right\rangle=\sum_{m=1}^{M} P_{m} c_{\mathrm{mig}, m}$.

To analyze the effect of wave and wind conditions, we also calculate the dynamics of the system with tide only $\left(\omega_{\text {tide }}, c_{\text {mig,tide }}\right)$ i.e. no waves and wind: $U_{\text {wind }}=0$ and $U_{\text {orb }}=0$. For each bin, we then define the probability-weighted relative growth and migration rate according to

$\Delta \omega_{m}=P_{m}\left(\omega_{m}-\omega_{\text {tide }}\right)$,

$\Delta c_{\mathrm{mig}, m}=P_{m}\left(c_{\mathrm{mig}, m}-c_{\mathrm{mig}, \mathrm{tide}}\right)$
The probability-weighted storm effect gives insight which storm conditions have the biggest influence on the total climate-averaged sand wave dynamics.

\section{Results}

\subsection{Probability density function}

The probability density function resulting from the binning procedure is in fact a four dimensional matrix, which is visualized in Fig. 3. The distinct patterns in the colored part of the figure (top) indicate that wave and wind conditions are correlated, most clearly regarding direction. The peaks in the bar plots (bottom row) show the dominant wind speed (around 5-10 m/s), wind direction (roughly SW), orbital velocity $(0-0.05 \mathrm{~m} / \mathrm{s})$ and wave direction (also SW). Also many of the bins are empty or close to empty showing that those conditions never or hardly occur. The probability density function shows that extreme wave and wind conditions rarely occur, waves hardly reach significant orbital velocities for most of the time. Larger wave orbital velocities occur mainly in combination with larger wind speeds, and the wave and wind direction are strongly correlated suggesting that most of the measured waves are locally induced by the wind conditions.

\subsection{Wave and wind climate-averaged dynamics}

Fig. 4 shows for both fair-weather and wave and wind climateaveraged conditions the growth and migration rate as function of $\left(k_{x}, k_{y}\right)$. For fair-weather conditions the FGM is a mode with crests perpendicular to the tidal flow direction and with a wavelength of $L_{\mathrm{FGM}}=356 \mathrm{~m}$, the growth rate is $\omega_{\mathrm{FGM} \text {, tide }}=0.390 \mathrm{yr}^{-1}$ and due to the symmetrical tidal forcing the migration rate is zero.

In comparison, for the wave and wind-climate averaged results, the FGM has hardly shifted: it has a wavelength of $\left\langle L_{\mathrm{FGM}}\right\rangle=361 \mathrm{~m}$, a growth rate of $\left\langle\omega_{\mathrm{FGM}}\right\rangle=0.387 \mathrm{yr}^{-1}$ and a migration rate of $\left\langle c_{\mathrm{mig}, \mathrm{FGM}}\right\rangle=6.6 \mathrm{~m} \mathrm{yr}^{-}$ ${ }^{1}$. A positive migration rate is in the direction of the positive $x$-axis, as shown in Fig. 1.

\subsection{Storm effects on growth and migration rate}

The four-dimensional relative growth and migration rates, corresponding to the climate-averaged fastest growing mode (denoted with a plus-sign in Fig. 4c-f) are shown in Figs. 5 and 6, respectively.

Fig. 5 shows both negative and positive growth rate contributions (relative to fair-weather) indicating that storm conditions can both enhance and reduce sand wave growth. Adding all growth rate contributions results in a rather small overall negative contribution to the growth rate of $\Delta \omega=\langle\omega\rangle-\omega_{\text {tide }}=-2.9 \cdot 10^{-3} \mathrm{yr}^{-1}$. These negative values also appear for other topographic wave numbers $\left(k_{x}, k_{y}\right)$, as seen from the growth rate plots in Fig. 4e. Regarding migration rates, shown in Fig. 6, we observe predominantly positive contributions from the southwest direction.

\subsection{Mild versus extreme conditions}

To address the question which storm conditions have the largest effects mild, medium and extreme conditions are defined. This classification is based on wave orbital velocity and wind speed values that coincide with bin bounds, and irrespective to wave or wind angle. First, 'mild' conditions are considered to have wave orbital velocities below $0.05 \mathrm{~m} / \mathrm{s}$ and wind speeds below $8.33 \mathrm{~m} / \mathrm{s}$. Then, 'medium' conditions have orbital velocities below $0.1 \mathrm{~m} / \mathrm{s}$ and wind speeds below $16.67 \mathrm{~m} / \mathrm{s}$. The remaining conditions are termed 'extreme'. Mild, medium and extreme conditions are indicated by the colors in Fig. 7. Fig. 7a shows the percentages of the probability of mild, medium and extreme conditions to occur and 7(b) shows the relative to fair-weather migration rate contribution. Note that for computing the percentages, the absolute 

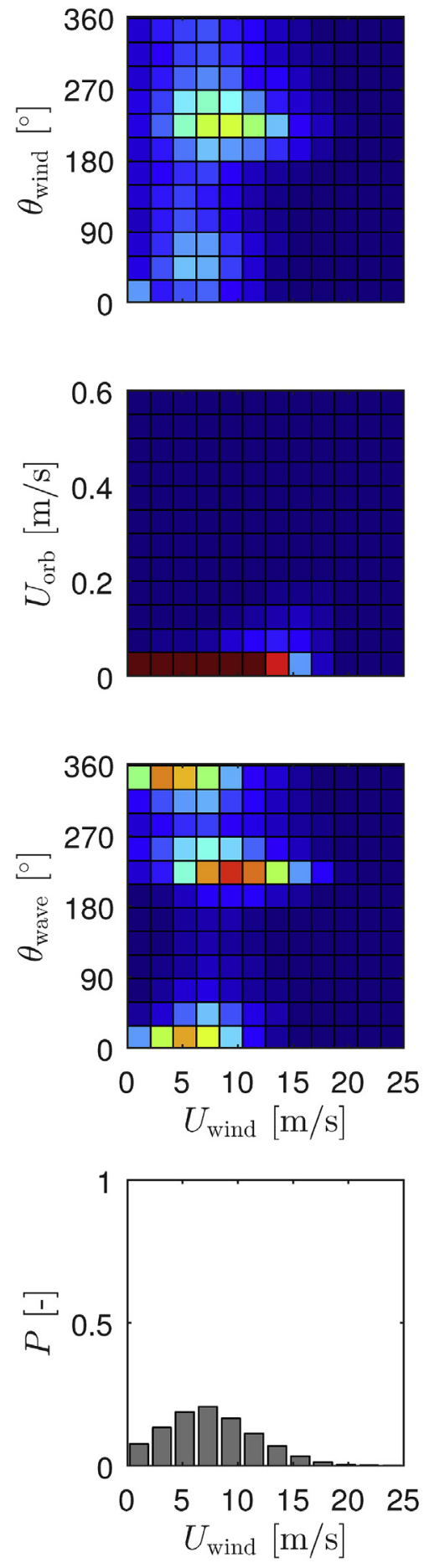
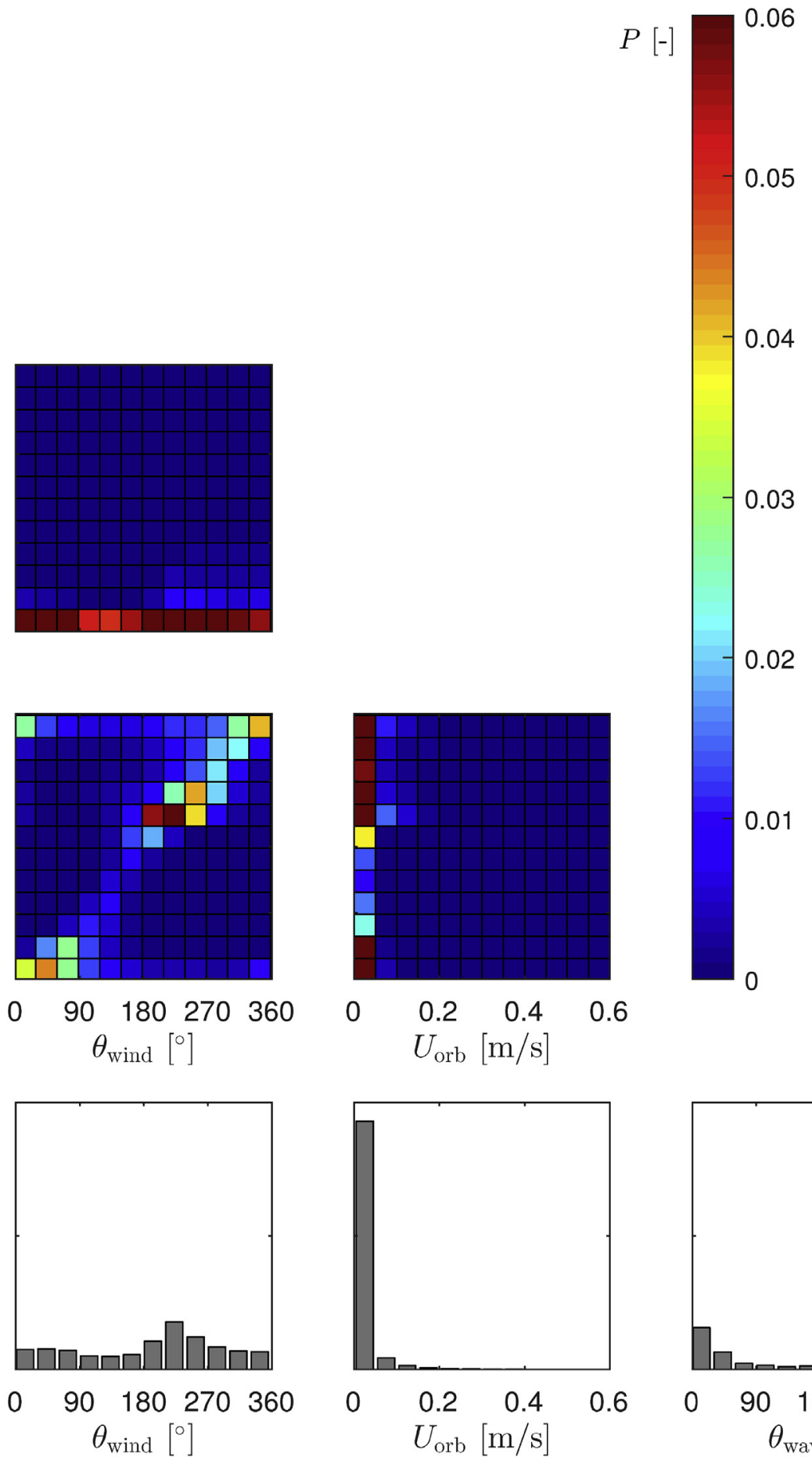

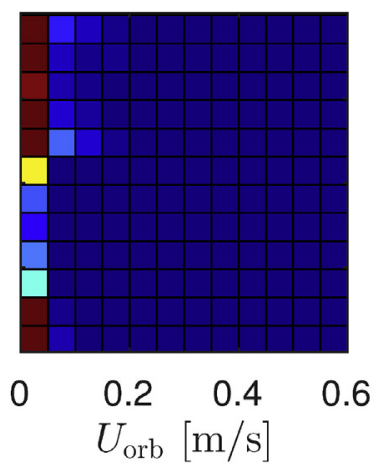

0.02

0.04

0.03

0.01

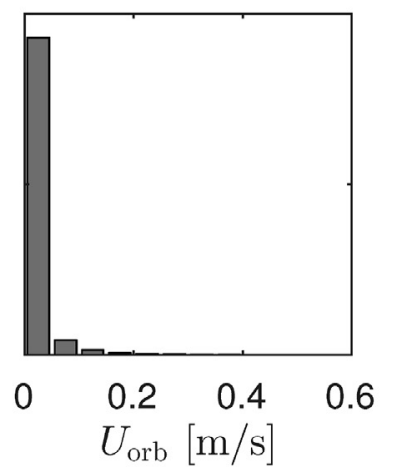

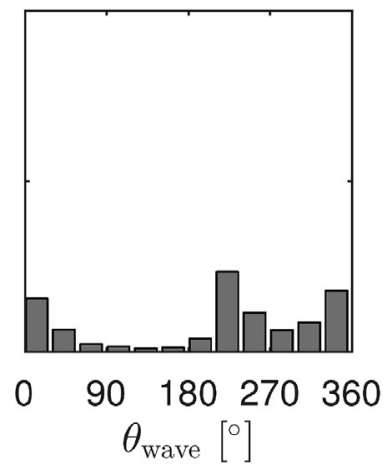

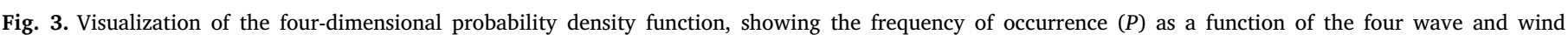

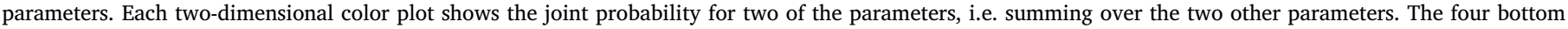

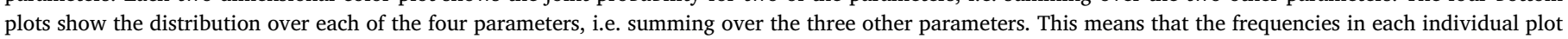
add to one. (For interpretation of the references to color in this figure legend, the reader is referred to the Web version of this article.)

value of the relative to fair-weather growth and migration rate of each bin has been taken.

The plots in Fig. 7 clearly show a shift in percentages between the probability contribution and the migration rate contribution. Whereas mild conditions occur for $59.7 \%$ of the time, they result in only a contribution of $17 \%$ to the total migration rate. Intermediate conditions occur for $36.8 \%$ of the time, whereas they cause $67 \%$ of the total migration rate. Extreme conditions occur for $3.5 \%$ of the time, causing $15.7 \%$ of the total migration rate. These percentages of course depend on the definition of mild, medium and extreme conditions, but the general picture remains the following. Relative to their occurrence, the contribution of mild conditions to the migration rate is only small. For medium conditions the contribution to the migration rate is large compared to their occurrence. This shift is even stronger for extreme conditions. Overall, the major contribution to sand wave migration is caused by conditions that combine a sufficiently large effect with a relatively large probability of occurrence.

The contributions of mild, medium and extreme conditions to $\Delta \omega$ 

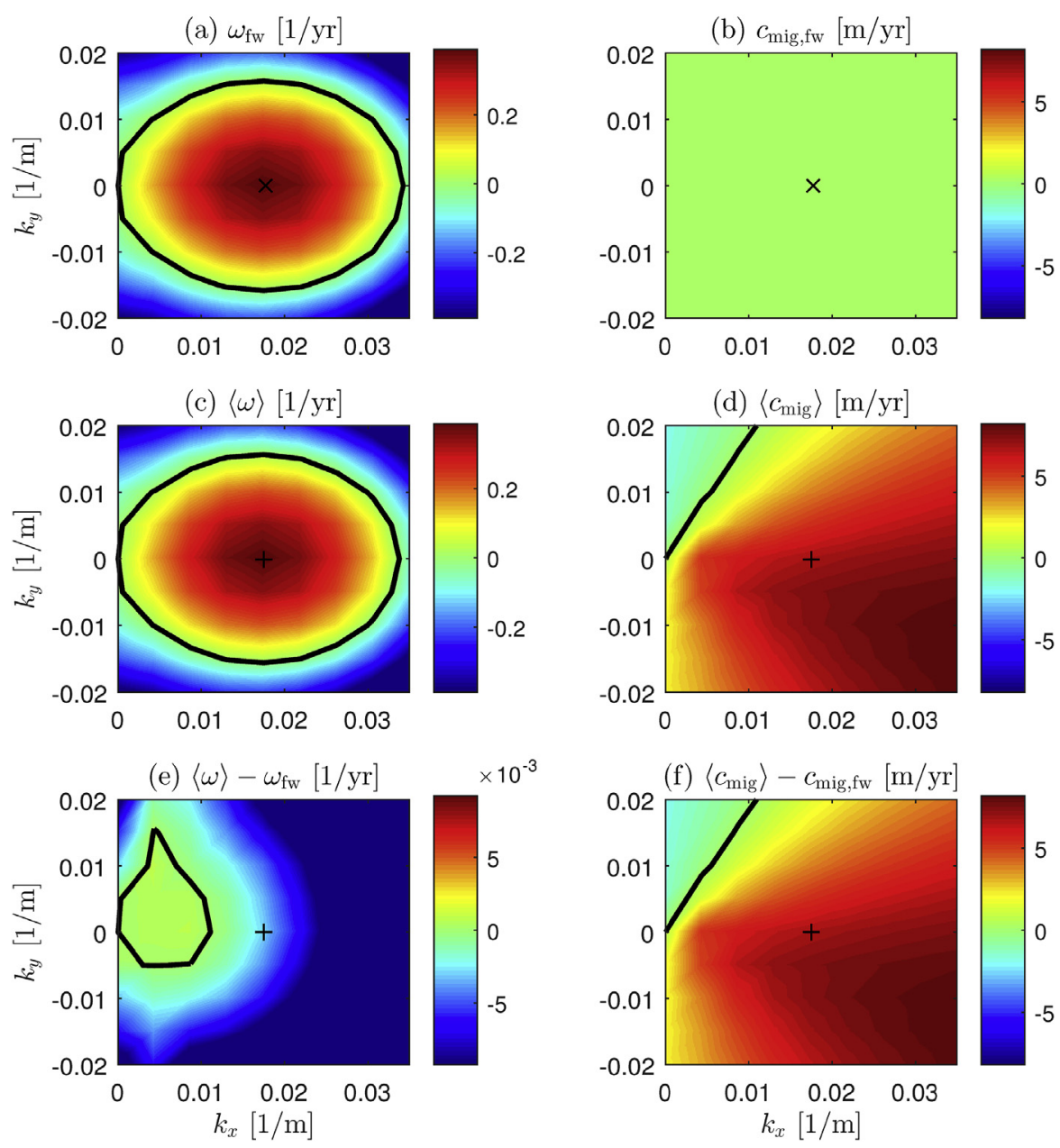

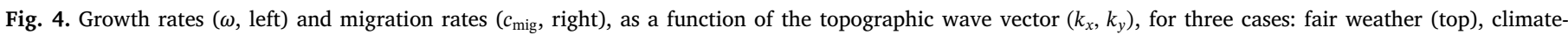
averaged (middle), difference (bottom). The $\times$ and + indicate the fair-weather and climate averaged fastest growing modes, respectively.

show a similar shift (not shown here). Because the total effect on the climate-averaged growth rate is small (Section 3.3), we do not further analyze this.

\subsection{Seasonal variations: winter vs summer}

By repeating our analysis for yearly seasonal sections of the wave and wind data we obtain seasonal wave and wind averaged FGM properties. Here we defined the winter season for a given year as from 1 October of the past year until 1 March. The summer season is from 1 March until the 1 October. These FGM properties as function of year are shown in Fig. 8.

Here we observe that especially the migration rate shows large seasonal variations, which support variation of observed migration due to storm effects (Fenster et al., 1990) as well as seasonal variations (Harris, 1989). The mean migration rate in summer is $3.4 \mathrm{~m} / \mathrm{yr}$ (with standard deviation $\sigma=1.7 \mathrm{~m} / \mathrm{yr})$ and $8.1 \mathrm{~m} / \mathrm{yr}$ in winter $(\sigma=2.2 \mathrm{~m}$ / yr).

Also the growth rate and the wavelength of the fastest growing mode show a systematic seasonal variation, be it only small. The mean growth rate in summer is $0.392 \mathrm{yr}^{-1}\left(\sigma=2.3 \cdot 10^{-3} \mathrm{yr}^{-1}\right)$ and $0.385 \mathrm{yr}^{-1}$ in winter $\left(\sigma=3.2 \cdot 10^{-3} \mathrm{yr}^{-1}\right)$. The mean wavelength in summer is $355 \mathrm{~m}$ $(\sigma=2.1 \mathrm{~m})$ and $363 \mathrm{~m}$ in winter $(\sigma=2.9 \mathrm{~m})$.

\subsection{Comparison with field observations}

Menninga (2012) analyzed sand wave characteristics at various locations in the North Sea. Close to the Euro Platform he analyzed a sand wave transect with 15 surveys between 1992 and 2010. From 1996 onwards we have both wave and wind data from the Euro Platform (Fig. 2). Here, we compare the migration rates obtained from our model approach using the wave and wind data between the sand wave survey moments with observed migration rates from the analysis of Menninga (2012). As shown in Fig. 9, we find a correlation between modeled and observed migration rates, which can be captured in a linear function fitted using the least squares reads:

$c_{\text {mig,obs }}=-4.04+0.817 c_{\text {mig,model }}$.

with correlation coefficients $R=0.67$ and $p=0.035$. This shows a reasonable agreement indicating that the method might work to estimate sand wave migration based on wave and wind observations. Nevertheless these statistics are based on only 10 data points which is certainly not sufficient to claim that the method is robust. The coefficients in Eq. (12) are not by any means universal and should be determined for a specific location. The modeled migration overestimates the observed values and the constant -4.04 in Eq. (12) suggests a tidal asymmetry or residual current, which we did not include in our model.

\section{Discussion}

Our model results have shown that for a simple setup, we have obtained realistic values of sand wave migration rate and wavelength. The following simplifications have been made: symmetrical tidal current, no Coriolis effect and bed load transport only. Observational data 

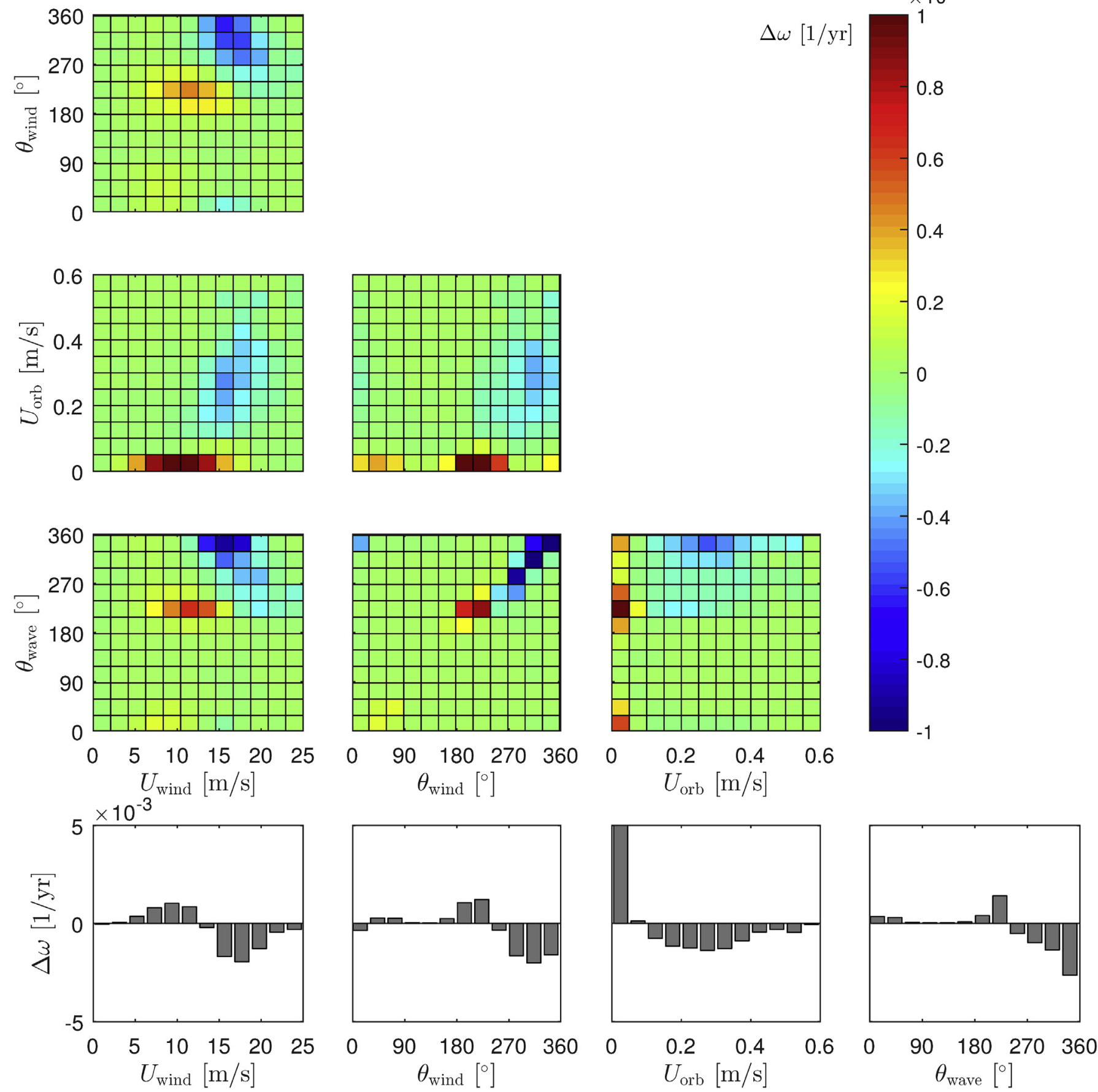

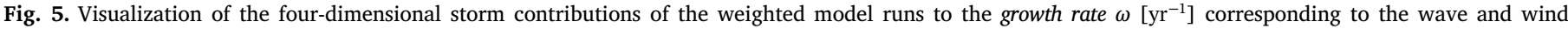

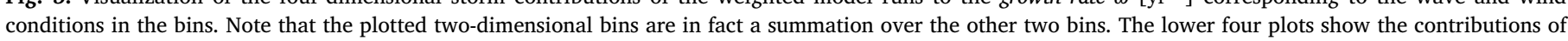
each of the four wave and wind conditions. These growth rate contributions correspond to the climate-averaged FGM.

show (Le Bot et al., 2000, Fig. 4) that veering of the flow due to the Coriolis effect is not as large as predicted in our infinitely large model domain. This justifies neglecting the Coriolis effect in our simulations. The effect of suspended load sediment transport is left out in our analysis as environmental conditions at the study site show that bed load is the dominant transport mode (see Fig. 8A in Damen et al., 2018).

The turbulence model used in the model is based on a constant eddy viscosity which reasonably resolves the flow outside the boundary layer, supplemented with a partial slip condition at the seabed to simulate smaller turbulent mixing near the bed to find reasonable shear stresses at the seabed. The results of the linear stability sand wave model turn out to be sensitive to this partial slip parameter. Other studies investigated the use of more sophisticated turbulence models in sand wave modeling, and show that quantitatively more refined turbulence modeling improves sand wave generation. However, more refined turbulence models come at the cost of more computational effort. Qualitatively a constant eddy viscosity model is able to capture the generating mechanism of sand waves, if the partial slip parameter is properly chosen. We tuned the partial slip parameter such that in fairweather conditions the sand wave wavelength corresponds to those observed in the field. The constants determined in Eq. (12) are partially explained by the simplifications in the setup. However, the model is still 

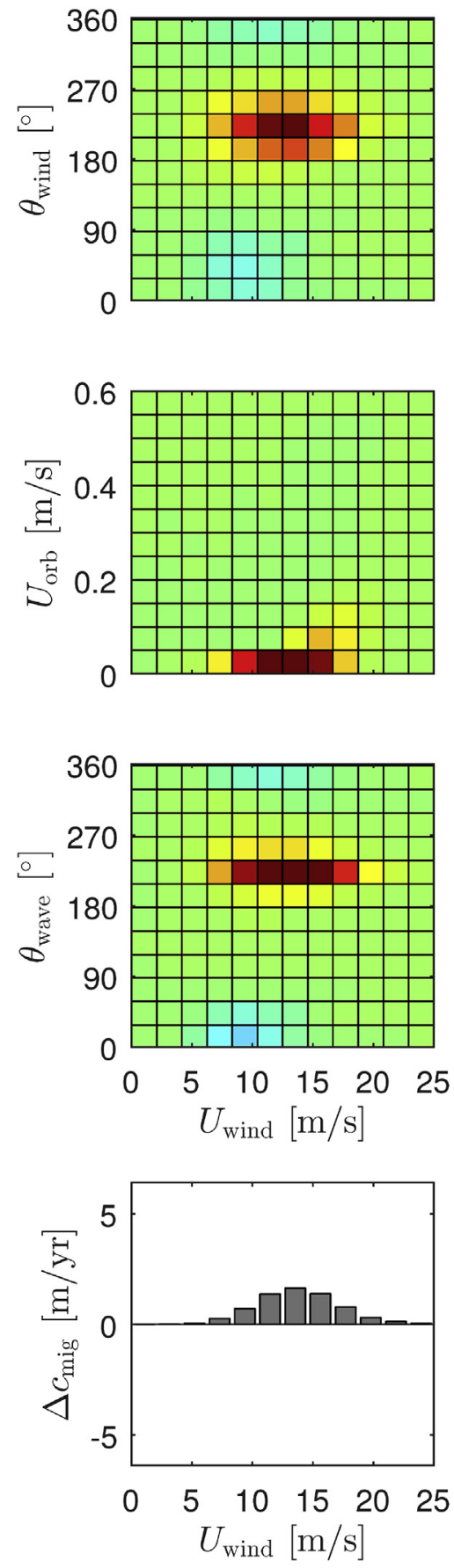
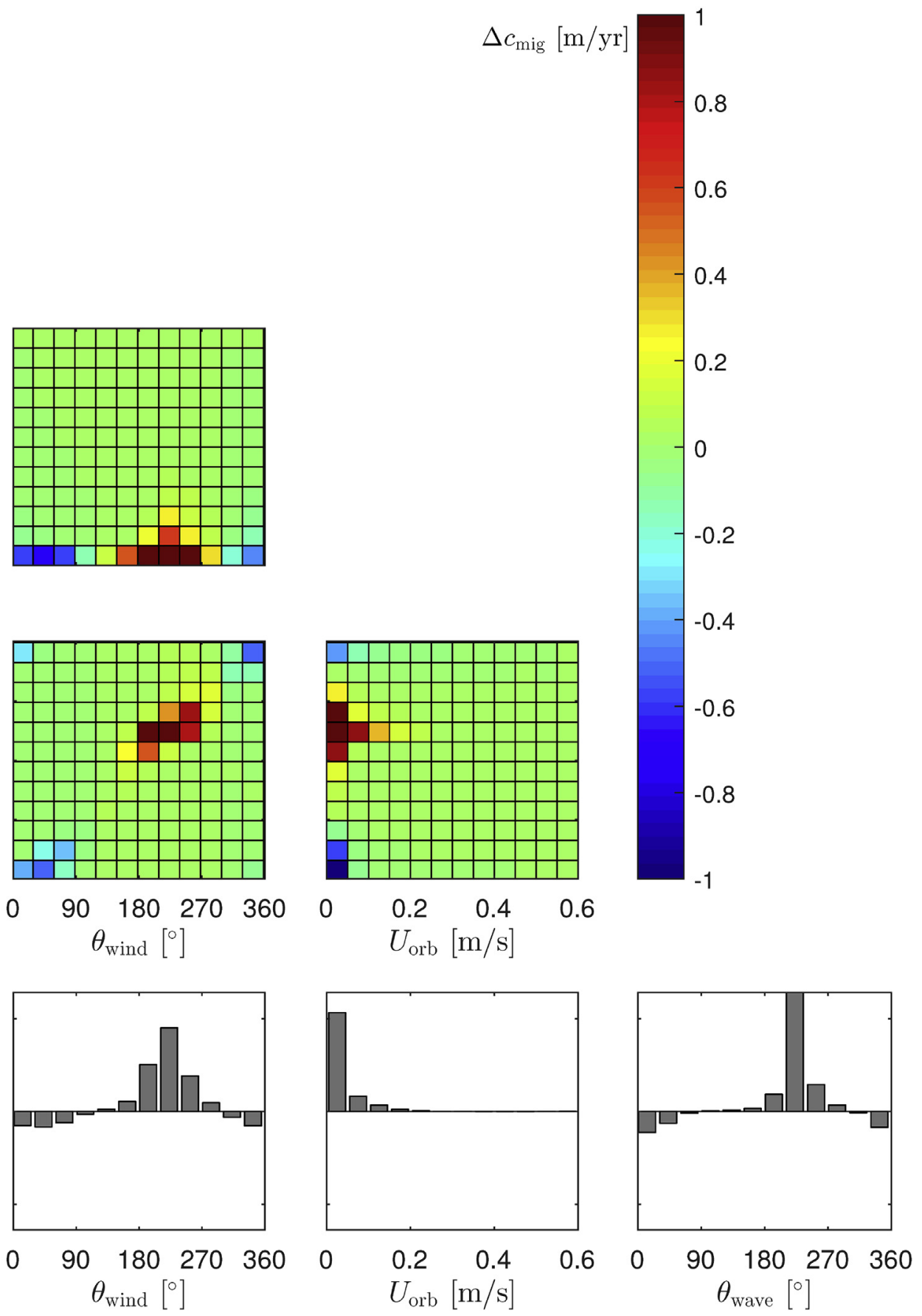

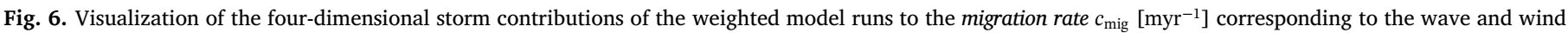

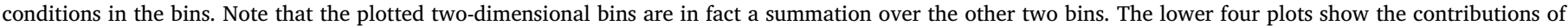
each of the four wave and wind conditions. These migration rate contributions correspond to the climate-averaged FGM.

able to capture the most important dynamics.

As it turns out that variations in wave and wind conditions also lead to variations in sand wave migration our model could prove useful as a predictive tool solely based on measured wave and wind conditions, potentially decreasing the bathymetric surveying frequency that is required to guarantee shipping safety.

In other locations variations in sand wave migration have been observed, and these are associated with variations in wind conditions (Le Bot et al., 2000; Krewinkel et al., 2017). In the Adolphus Channel, northeast of Australia, Harris (1989) shows seasonal reversal of sand wave migration during the monsoon season.

Since we make use of a linear stability model, for which we look at the formation stage of small sand waves, the sequence in which wave and wind conditions occur is irrelevant. For large sand wave amplitudes a nonlinear analysis is required, in which the sequence in which wave and wind conditions occur is relevant. However, an equivalent nonlinear analysis with equally many wave and wind conditions is not yet feasible with available sand wave models.

Our model findings imply that climate change will affect sand wave dynamics. If waves and wind conditions become more extreme, this will result in larger sand wave migration rates.

Our work shows that wind and waves can cause a seasonal varying effect on sand wave characteristics. Particularly the migration rate is subject to seasonal variations. The change in preferred wavelength is 

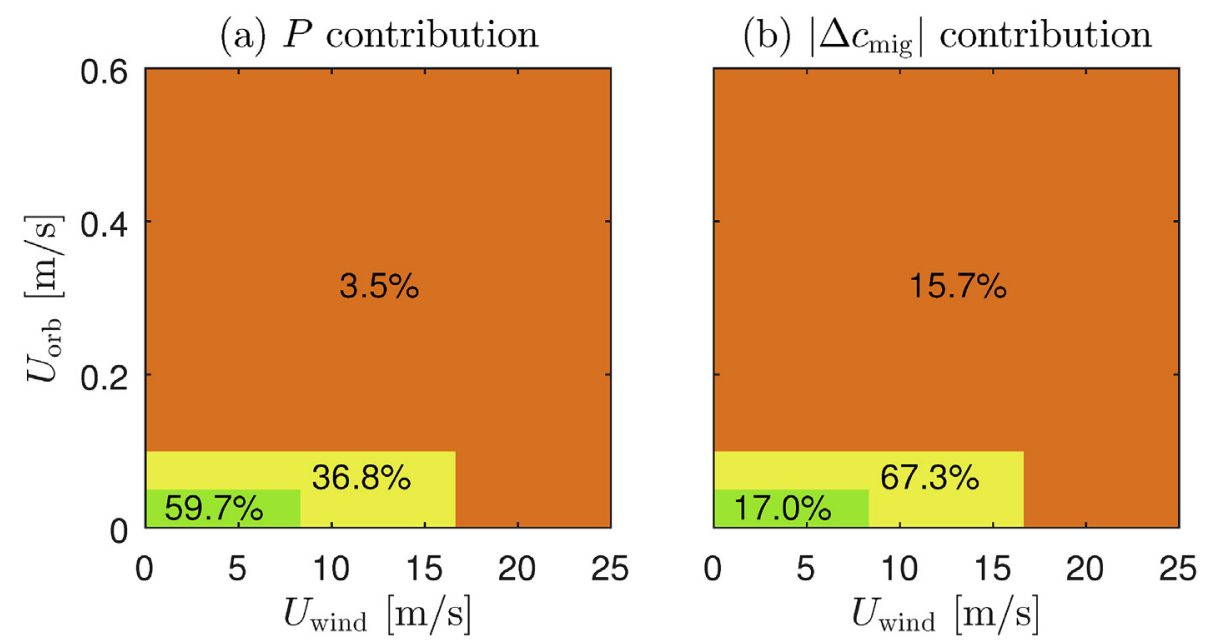

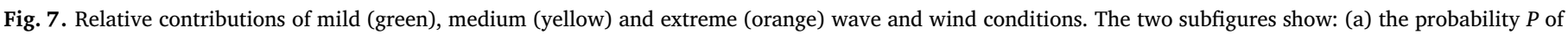

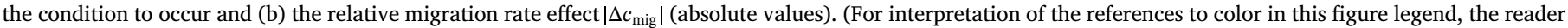
is referred to the Web version of this article.)

only small, and on the timescale of seasonal variations sand waves are unlikely to show variations in wavelength. Furthermore it should be noted that various other phenomenon may affect sand waves seasonally, such as biology (Borsje et al., 2009; Damveld et al., 2017) or thermally induced variations in tidal characteristics (Gräwe et al., 2014).

Blondeaux and Vittori (2016) includes a wide variety of processes in their model. Among them wind waves, the spring-neap cycle and a residual tidal component. In our work we focus in detail on an actual wave and wind climate on sand wave dynamics, while using a more simplified version of other processes. Our work agrees with their model findings and additionally shows that seasonal variations may occur as well as which of the wave and wind conditions contribute the most.

This research has been carried out as part of a bigger project called SMARTSEA. The goal of the project is to improve the efficiency and safety of navigation. For safe navigation in shallow seas such as the North Sea with a dynamic seabed, mainly caused by sand wave dynamics, accurate nautical charts are required. At present sand wave dynamics are poorly predictable and frequent surveying is required. This research shows from a process-based perspective that storms are one of the mechanisms that causes the irregularities in sand wave dynamics. A better understanding of the temporally varying migration
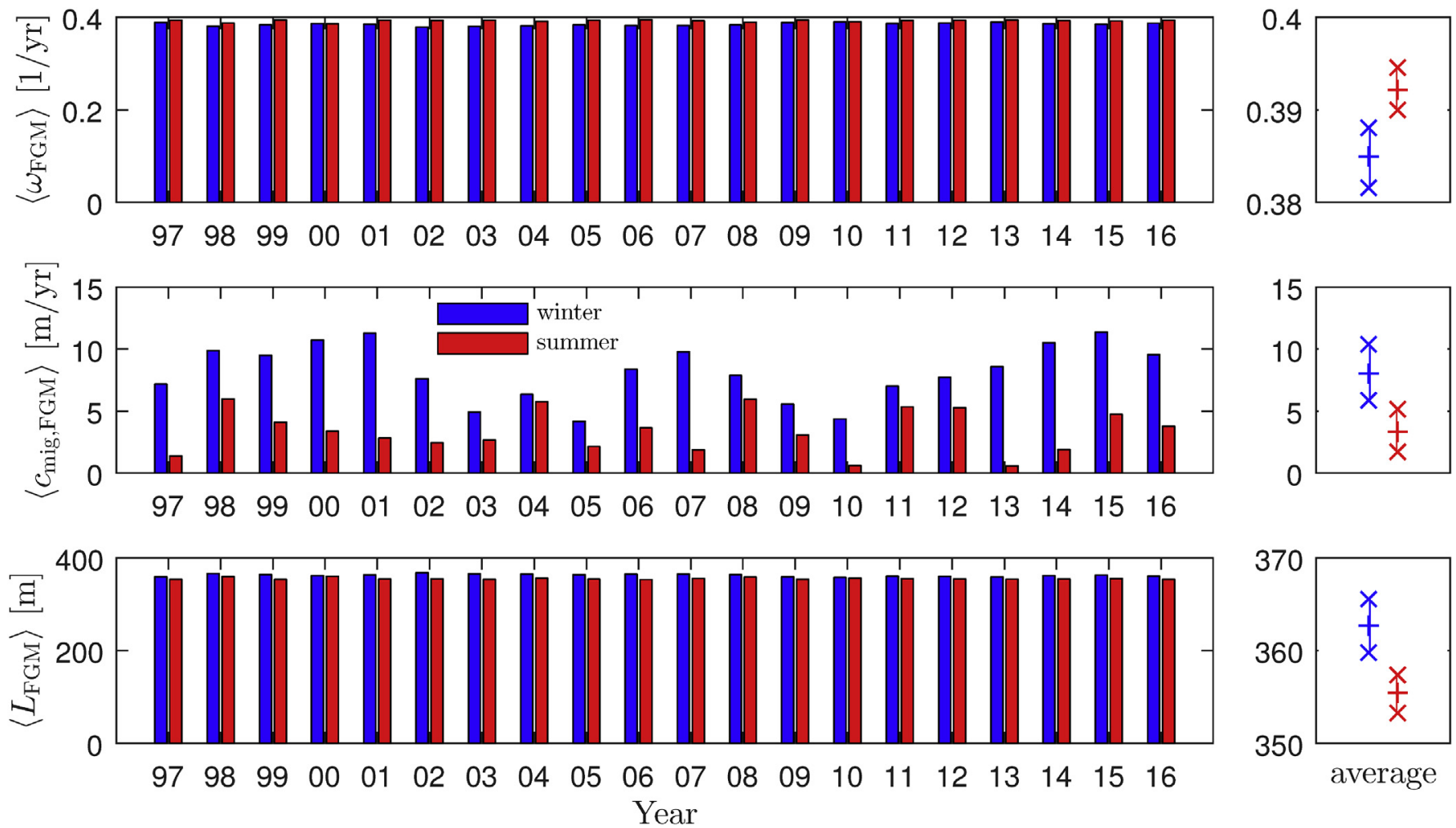

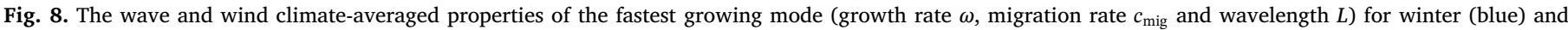

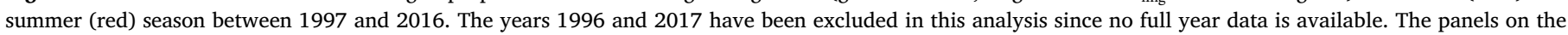

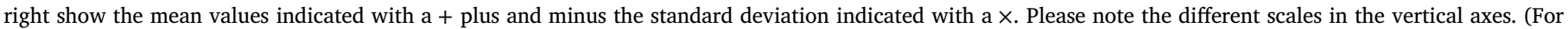
interpretation of the references to color in this figure legend, the reader is referred to the Web version of this article.) 
(a)

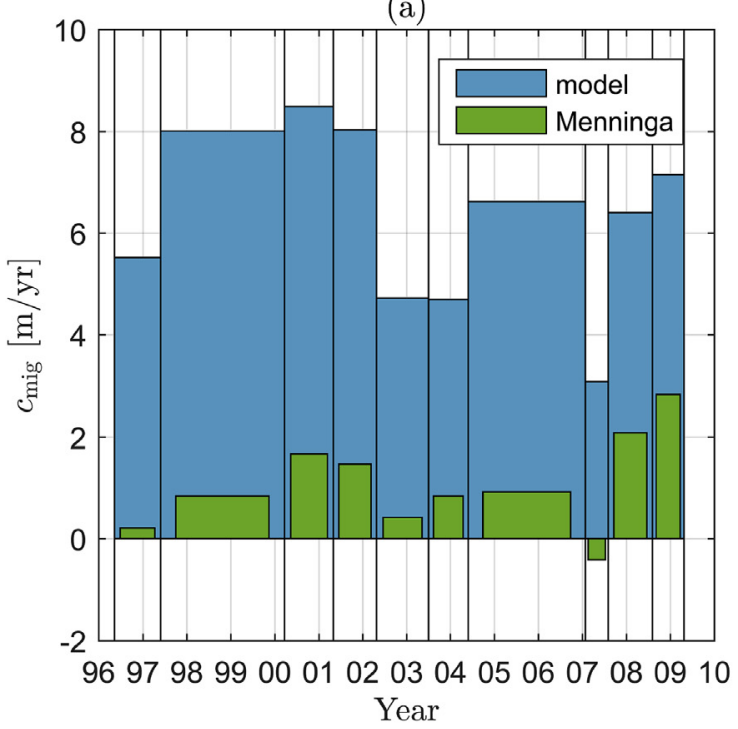

(b)

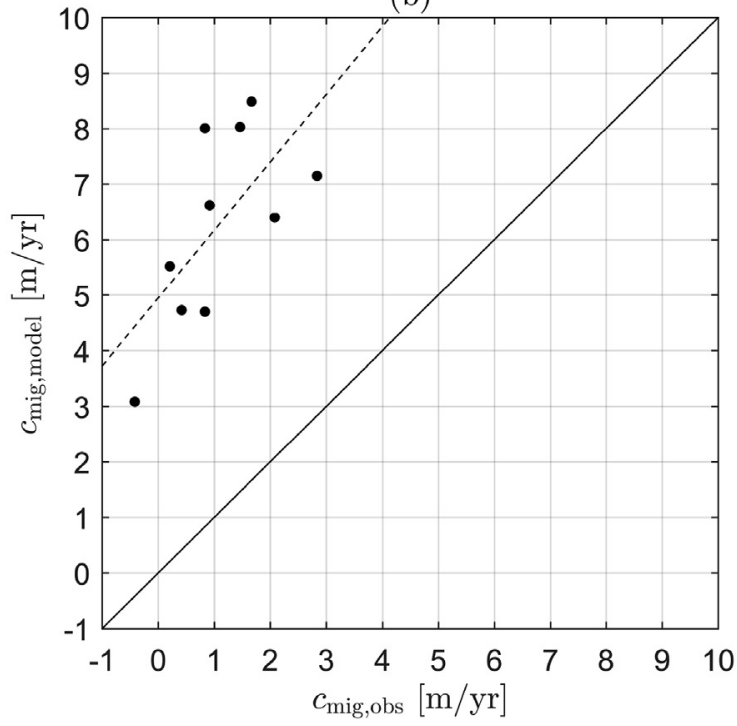

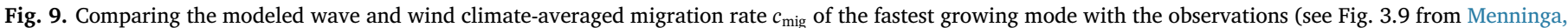

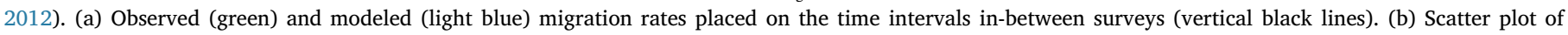

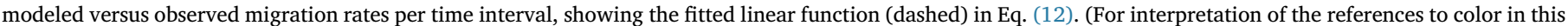
figure legend, the reader is referred to the Web version of this article.)

and growth behavior of sand wave dynamics will eventually reduce the surveying frequencies and lead to more efficient maintenance strategies for navigation channels.

Ernstsen et al. (2011) observed spatiotemporal variations in sand wave height in the Grådyb tidal inlet channel (Denmark) and linked this to a nearby scour hole formed by a sunk dredger. In their work they observe temporal variations in sand wave height and report a large influence of the scour hole on the sand wave height. Before the formation of the scour hole temporal variations in height are also present, albeit that these variations are smaller than the variations after the formation and subsequent filling of the scour hole. In our work we were restricted to small-amplitude dynamics. Wind and waves may well be able to cause temporally variable sand wave height variations in a similar fashion as the observed migration rate. This would require future research.

\section{Conclusion}

By combining wind and wave data with an idealized linear stability model we were able to investigate the effect of an actual storm climate on sand wave dynamics near the Euro Platform in the North Sea. We find that storms affect particularly migration, and to a smaller extent the growth rate. The fastest growing mode properties of sand waves in a storm climate of the past 20 years have a slightly lower growth rate and a slightly longer wavelength, compared to the fair-weather model results. The most notable effect is a $6.6 \mathrm{~m} \mathrm{yr}^{-1}$ migration rate towards the northeast. The very small $(\sim 1 \%)$ decrease in growth rate indicates that the growth and wavelength of sand waves is hardly affected by storm effects at our study site.

Because the model is quick, it enables to conduct a very large number of simulations (order $10^{4}$ ). We have identified the type of wind and wave conditions with the largest contributions, taking into account both the probability of occurrence and their effect. The analysis showed that even though some conditions rarely occur they still had a prominent contribution due to their large effect during such conditions. We find that medium conditions are responsible for two thirds of the migration rate $(\sim 67 \%)$, while these conditions occur roughly only one third of the time $(\sim 37 \%)$.

Partitioning the data sets of wind and wave conditions into yearly seasonal time series allowed us to investigate the effect of seasonal effects. We observed a clear trend between summer and winter periods. Typically sand wave migration rates are larger during winter times compared to the more calm summer periods. Similarly growth rates are lower and the preferred wavelength is larger during winter periods compared to the summer periods, although these effects are only small compared to migration rate effects. Migration rate variations have been observed by many observational studies, for which many of the authors referred to the potential effects of storms. From a process-based modeling perspective, we have linked migration rates of sand waves in the North Sea (and their seasonal fluctuations) to observed variations in wave and wind conditions. Finally, comparison of our model results with observed sand wave migration supports the suggested link between the occurrence of wave and wind conditions and temporal variability in sand wave migration.

\section{Acknowledgements}

This work is part of the research programme SMARTSEA with project number 13275, which is (partly) financed by the Netherlands Organisation for Scientific Research (NWO). The authors thank John Damen for providing site specific sand wave characteristics. We thank the reviewers Alice Lefebvre and Paolo Blondeaux for their comments.

\section{References}

Besio, G., Blondeaux, P., Brocchini, M., Vittori, G., 2004. On the modeling of sand wave migration. J. Geophys. Res.: Oceans 109 (1978-2012).

Blondeaux, P., Vittori, G., 2016. A model to predict the migration of sand waves in shallow tidal seas. Continent. Shelf Res. 112, 31-45.

Blondeaux, P., Vittori, G., Mazzuoli, M., 2016. Pattern formation in a thin layer of sediment. Mar. Geol. 376, 39-50.

Borsje, B.W., de Vries, M.B., Bouma, T.J., Besio, G., Hulscher, S.J.M.H., Herman, P.M.J. 2009. Modeling bio-geomorphological influences for offshore sandwaves. Continent. Shelf Res. 29, 1289-1301.

Campmans, G.H.P., Roos, P.C., De Vriend, H.J., Hulscher, S.J.M.H., 2017. Modeling the influence of storms on sand wave formation: a linear stability approach. Continent. Shelf Res. 137, 103-116.

Campmans, G.H.P., Roos, P.C., De Vriend, H.J., Hulscher, S.J.M.H., 2018. The influence of storms on sand wave evolution: a nonlinear idealized modeling approach. J. Geophys. Res.: Earth Surface. https://agupubs.onlinelibrary.wiley.com/doi/abs/10. 1029/2018JF004616.

Damen, J.M., Van Dijk, T.A.G.P., Hulscher, S.J.M.H., 2018. Spatially varying environmental properties controlling observed sand wave morphology. J. Geophys. Res.: 
Earth Surface 123, 262-280

Damveld, J.H., Roos, P.C., Borsje, B.W., Hulscher, S.J.M.H., 2017. Exploring the Two-way Coupling between Sand Wave Dynamics and Benthic Species: an Idealised Modelling Approach. 4th International Symposium on Shallow Flows, Eindhoven, Netherlands. Dodd, N., Blondeaux, P., Calvete, D., De Swart, H.E., Falqués, A., Hulscher, S.J.M.H., Różyński, G., Vittori, G., 2003. Understanding coastal morphodynamics using stability methods. J. Coast Res. 849-865.

Ernstsen, V.B., Lefebvre, A., Bartholdy, J., Bartholomä, A., Winter, C., 2011. Spatiotemporal height variations of large-scale bedforms in the grådyb tidal inlet channel (Denmark): a case study on coastal system impact. J. Coast Res. 746.

Fenster, M.S., Fitzgerald, D.M., Bohlen, W.F., Lewis, R.S., Baldwin, C.T., 1990. Stability of giant sand waves in eastern Long Island Sound, USA. Mar. Geol. 91, 207-225.

Gräwe, U., Burchard, H., Müller, M., Schuttelaars, H.M., 2014. Seasonal variability in M2 and M4 tidal constituents and its implications for the coastal residual sediment transport. Geophys. Res. Lett. 41, 5563-5570.

Harris, P.T., 1989. Sandwave movement under tidal and wind-driven currents in a shallow marine environment: Adolphus Channel, northeastern Australia. Continent. Shelf Res. 9, 981-1002.

Houthuys, R., Trentesaux, A., De Wolf, P., 1994. Storm influences on a tidal sandbank's surface (Middelkerke Bank, southern North Sea). Mar. Geol. 121, 23-41.

Hulscher, S.J.M.H., 1996. Tidal-induced large-scale regular bed form patterns in a threedimensional shallow water model. J. Geophys. Res.: Oceans 101, 20727-20744 (1978-2012).

Hulscher, S.J.M.H., Van den Brink, G.M., 2001. Comparison between predicted and observed sand waves and sand banks in the North Sea. J. Geophys. Res.: Oceans 106, 9327-9338 (1978-2012).

KNMI, n.d. www.knmi.nl,

Krewinkel, B.C., Straatsma, L., Dusseljee, D., Borsje, B.W., Hulscher, S.J.M.H., 2017. Sand Wave Migration Reversal Due to Severe Wind Events. NCK days. Den Helder, Netherlands 81-81.

Le Bot, S., Trentesaux, A., Garlan, T., Berne, S., Chamley, H., 2000. Influence des tempêtes sur la mobilité des dunes tidales dans le détroit du Pas-de-Calais. Oceanol. Acta 23,
$129-141$.

Mei, C.C., 1989. The Applied Dynamics of Ocean Surface Waves, vol. 2 World Scientific Publishing Co. Pte. Ltd.

Menninga, P.J., 2012. Analysis of Variations in Characteristics of Sand Waves Observed in the Dutch Coastal Zone: a Field and Model Study. Master Thesis. Utrecht University, NL.

Németh, A.A., Hulscher, S.J.M.H., De Vriend, H.J., 2002. Modelling sand wave migration in shallow shelf seas. Continent. Shelf Res. 22, 2795-2806.

Németh, A., Hulscher, S.J.M.H., De Vriend, H.J., 2003. Offshore sand wave dynamics, engineering problems and future solutions. Pipeline Gas J. 230, 67-69.

Németh, A.A., Hulscher, S.J.M.H., Van Damme, R.M.J., 2007. Modelling offshore sand wave evolution. Continent. Shelf Res. 27, 713-728.

Rijkswaterstaat, n.d. www.live.waterbase.nl.

Roos, P.C., Hulscher, S.J.M.H., Van der Meer, F., Van Dijk, T.A.G.P., Wientjes, I.G.M., Van den Berg, J., 2007. Grain Size Sorting over Offshore Sandwaves: Observations and Modelling.

Terwindt, J.H.J., 1971. Sand waves in the southern bight of the North sea. Mar. Geol. 10, $51-67$.

Van den Berg, J., Sterlini, F., Hulscher, S.J.M.H., Van Damme, R.M.J., 2012. Non-linear process based modelling of offshore sand waves. Continent. Shelf Res. 37, 26-35.

Van Dijk, T.A.G.P., Kleinhans, M.G., 2005. Processes controlling the dynamics of com pound sand waves in the North Sea, Netherlands. J. Geophys. Res.: Earth Surface 110 (2003-2012).

Van Gerwen, W., Borsje, B.W., Damveld, J.H., Hulscher, S.J.M.H., 2018. Modelling the effect of suspended load transport and tidal asymmetry on the equilibrium tidal sand wave height. Coast Eng. 136, 56-64.

Van Oyen, T., Blondeaux, P., 2009. Grain sorting effects on the formation of tidal sand waves. J. Fluid Mech. 629, 311-342.

Van Santen, R.B., De Swart, H.E., Van Dijk, T.A.G.P., 2011. Sensitivity of tidal sand wavelength to environmental parameters: a combined data analysis and modelling approach. Continent. Shelf Res. 31, 966-978. 